\title{
Fear of hypoglycaemia: defining a minimum clinically important difference in patients with type 2 diabetes Tom Stargardt*1,2, Linda Gonder-Frederick ${ }^{3}$, Karl J Krobot ${ }^{4}$ and Charles M Alexander ${ }^{5}$
}

\author{
Address: ${ }^{1}$ Health Services Management, Munich School of Management, Munich University, Germany, ${ }^{2}$ Institute of Health Economics and Health \\ Care Management, Helmholtz Zentrum Munich, Germany, ${ }^{3}$ Department of Psychiatry and Neurobehavioral Sciences, University of Virginia, \\ Charlottesville, VA, USA, ${ }^{4}$ Outcomes Research, MSD Sharp \& Dohme GMBH, Haar, Germany and ${ }^{5}$ Outcomes Research, Merck \& Co, Inc, \\ Whitehouse Station, NJ, USA \\ Email: Tom Stargardt* - Stargardt@bwl.Imu.de; Linda Gonder-Frederick - LAG3G@hscmail.mcc.virginia.edu; \\ Karl J Krobot - karl_krobot@msd.de; Charles M Alexander - charles_alexander@merck.com \\ * Corresponding author
}

Published: 22 October 2009

Health and Quality of Life Outcomes 2009, 7:91 doi:10.1186/1477-7525-7-91

This article is available from: http://www.hqlo.com/content/7///9I

(C) 2009 Stargardt et al; licensee BioMed Central Ltd.

This is an Open Access article distributed under the terms of the Creative Commons Attribution License (http://creativecommons.org/licenses/by/2.0), which permits unrestricted use, distribution, and reproduction in any medium, provided the original work is properly cited.
Received: 3 June 2009

Accepted: 22 October 2009

\begin{abstract}
Background: To explore the concept of the Minimum Clinically Important Difference (MID) of the Worry Scale of the Hypoglycaemia Fear Survey (HFS-II) and to quantify the clinical importance of different types of patient-reported hypoglycaemia.

Methods: An observational study was conducted in Germany with 392 patients with type 2 diabetes mellitus treated with combinations of oral anti-hyperglycaemic agents. Patients completed the HFS-II, the Treatment Satisfaction Questionnaire for Medication (TSQM), and reported on severity of hypoglycaemia. Distribution- and anchor-based methods were used to determine MID. In turn, MID was used to determine if hypoglycaemia with or without need for assistance was clinically meaningful compared to having had no hypoglycaemia.

Results: I I 2 patients (28.6\%) reported hypoglycaemic episodes, with I 5 patients (3.8\%) reporting episodes that required assistance from others. Distribution- and anchor-based methods resulted in MID between 2.0 and 5.8 and 3.6 and 3.9 for the HFS-II, respectively. Patients who reported hypoglycaemia with (2I.6) and without (I2.I) need for assistance scored higher on the HFS-II (range 0 to 72 ) than patients who did not report hypoglycaemia (6.0).

Conclusion: We provide MID for HFS-II. Our findings indicate that the differences between having reported no hypoglycaemia, hypoglycaemia without need for assistance, and hypoglycaemia with need for assistance appear to be clinically important in patients with type 2 diabetes mellitus treated with oral anti-hyperglycaemic agents.
\end{abstract}

\section{Background}

The goal of treatment of diabetes is to achieve glycemic control in order to prevent long-term micro- and macrovascular complications. Due to the progressive nature of beta cell failure in spite of treatment with anti-hyperglycemic agents, $\mathrm{HBA}_{1 \mathrm{c}}$ levels slowly rise even after an initial fall in patients with type 2 diabetes [1-3]. Therefore an increasing number of patients eventually need to be 
treated with combination medication regimens and/or insulin $[4,5]$.

One of the major challenges in the treatment of diabetes is to achieve glycemic control while avoiding episodes of hypoglycaemia [6-8]. Hypoglycaemia is due to excess insulin levels in relation to circulating glucose. Etiologies may include missed meals, physical activity, drug interactions, or the anti-hyperglycaemic medication regimen [6,7,9-12]. If untreated, hypoglycaemia may affect brain function, both cognitive and motor. With severe hypoglycaemia, convulsions, coma or death may occur $[13,14]$. Recurrent severe episodes of hypoglycaemia can lead to behavioral changes [6], cognitive impairment [15], and unawareness of hypoglycaemia [16]. Because of these negative consequences, patients may develop psychological fear of hypoglycaemia. This fear can become phobic [17], reduce quality of life [18], and impact adherence with diabetes management $[7,12,13]$. Fear of hypoglycaemia decreases after interventions such as behavioral programs and islet cell transplant surgery [19-21].

However, little is known about fear of hypoglycaemia in patients with type 2 diabetes treated with combinations of oral agents. This study therefore explores the concept of the Minimum Clinically Important Difference (MID) for the Worry Scale of the Hypoglycaemia Fear Survey (HFSII) in a large sample of patients with type 2 diabetes treated with combinations of metformin and a sulphonylurea or metformin and a glitazone. According to the definition of Jaeschke, et al., MID is "the smallest difference in score [in the domain of interest] which patients perceive as beneficial and which would mandate [in the absence of troublesome side-effects and excessive cost] a change in the patient's management" [22]. As even very small absolute differences in patient-related outcomes such as HFS-II can become statistically significant given large group sizes, it is very important to find a threshold that indicates whether a difference in score is clinically meaningful or not [23].

This is the first study to develop a MID for fear of hypoglycaemia. The MID was then used to quantify and evaluate difference in HFS-II scores between patients who reported no hypoglycaemic episodes, hypoglycaemic episodes without need for assistance, and hypoglycaemic episodes with need for assistance.

\section{Methods}

\section{Setting and design}

An observational multi-centre study was conducted in Germany. Patients were recruited in a convenience sample of 92 sites by their physicians, either GPs or diabetologists. Data were collected between October and December 2005. To be included, patients were required to be diag- nosed with type 2 diabetes, 35 years or older, and had to be treated during the 6 months prior to the study with either a combination of metformin and a glitazone, or with a combination of metformin and a sulphonylurea. Patients were not eligible if they had been treated with insulin in the past, were taking part in a clinical trial, or were being treated for HIV or hepatitis. Data were collected using medical records review and questionnaires. Physicians were asked to provide information on the participant's medical history, baseline laboratory measures, and diabetes medications used during the six months prior to the study. After informed consent, participants completed the Treatment Satisfaction Questionnaire for Medication version 1.4 (TSQM) [24], a socio-demographic questionnaire, and the Worry Scale of the HFS-II. In addition, patients were asked about severity of hypoglycaemic episodes during the previous six months.

\section{Hypoglycaemia Fear Survey-II}

The HFS-II is a 33-item questionnaire with two subscales that measure 1) behaviours to avoid hypoglycaemia and its negative consequences and 2) worries about hypoglycaemia and its negative consequences. Responses are made on a 5-point Likert scale where $0=$ Never and $4=$ Always. This study used the 18 -item Worry subscale which has a score range of $0-72$ with higher scores indicating increased fear of hypoglycaemia. The HFS-II is a widely used measure in clinical trials, has been translated into more than 20 languages, and has demonstrated reliability and validity $[25,26]$.

\section{Construction of MID}

In general, studies have recommended using a variety of methods to determine MID $[27,28]$. In this study, we used a variety of established distribution-based and anchorbased methods. With regard to distribution-based methods, we used the score's standard error of measurement, the score's standard deviation multiplied by the square root of 1 minus Cronbach's alpha [29,30], and multiples of the score's standard deviation: $0.5,0.20,0.30$, and 0.33 $[23,27-29,31]$ to calculate MID. In addition, MID was computed as $8 \%$ of the theoretical score ranges (HFS-II: 72 points) [28].

As one of the patient-based anchors, we used treatment satisfaction to determine MID - due to its proximity to self-reported treatment success. Our MID is based on the assumption that patients who are not satisfied with their treatment are more likely to either not adhere to medications, or to complain to their doctors, which in turn may lead to a change in medications. We assume that treatment satisfaction is closely related to self-reported treatment success (i.e. 'worsening of condition' vs. 'no change in condition' or 'small improvement' vs. 'no change'), a concept that has been recommended to determine MID in 
many other studies $[27,28,32]$. Treatment satisfaction was measured as the response to the seven-point scaled TSQM question 14 'Taking all things into account, how satisfied or dissatisfied are you with this medication'. The answer categories that were less than 'satisfied' (e.g. 'somewhat satisfied', 'dissatisfied', 'very dissatisfied', and 'extremely dissatisfied') were combined as 'less than satisfied'. We thus determined MID for the Worry Scale of HFS-II by taking the difference in score means between patients who were 'satisfied' and patients who were 'less than satisfied' with their medication. As negative side effects, especially increased hypoglycaemia, may be also important and can lead to decreased medication adherence or a change in regimen, we also used responses to TSQM questions 6, 'To what extent do the side effects interfere with your physical health and ability to function', and TSQM question 8, 'To what degree have medication side effects affected your satisfaction with the medication', as anchors to determine MID. However, questions 6 and 8 of the TSQM were only answered by the smaller subsample of patients that experienced side-effects. Difference in means of the Worry Scale of HFS-II of patients who stated that they were 'somewhat' affected and patients that stated that they were 'quite a bit' or 'a great deal' affected were compared.

\section{Severity and fear of hypoglycaemia}

According to the recommendations of the American Diabetes Association [16], severity of hypoglycaemic episodes were categorized as 1 ) mild (little or no interruption of activities; no treatment assistance needed), 2) moderate (some interruption of activities; no assistance needed) and 3 ) severe (assistance of a third party needed). A fourth category, very severe hypoglycaemia, was added to capture episodes that required medical assistance. In the questionnaire, the different levels of severity of hypoglycaemia were defined for the patients who were asked if they experienced any of the four severity categories of hypoglycaemia during the last 6 month. If a patient reported episodes of hypoglycaemia at different levels of severity, the patient was classified according to the most severe episode reported. Following recommendations by other studies $[11,12,16,33]$, the categories mild and moderate were aggregated to 'hypoglycaemia without need for assistance' and the categories severe and very severe were aggregated to 'hypoglycaemia with need for assistance'. Results for MID were then applied to evaluate whether the difference between not having hypoglycaemic episodes and having different levels of severity of hypoglycaemic episodes is clinically important in this study population. In addition the mean scores of the Worry Scale of HFS-II for patients who reported hypoglycaemic episodes during the last six month were compared to patients who did not report hypoglycaemic episodes. One-way analysis of variance and Tukey's honestly significant differences test were used to test for statistical significance of differences in mean scores. A p-value of 0.05 was considered statistically significant. We also calculated Cohen's d statistics as a marker of effect size for differences in HFS-II. Statistical analyses were conducted using SAS version 9.1.3.

\section{Results}

From 402 patients recruited at 92 sites, two were excluded because they did not meet inclusion or exclusion criteria, and eight were excluded because they had not fully completed the Worry Scale of HFS-II. The final study population thus comprised 392 patients, of whom 268 patients were treated with metformin and a sulphonylurea, and 107 patients with metformin and a glitazone. For 17 patients it was unknown which of the two medication regimens they were on. As the differences between patients treated with metformin and a sulphonylurea and patients treated with metformin and a glitazone did not reach statistical significance for hypoglycaemia $(\mathrm{p}=0.1127)$ or HFS-II scores $(\mathrm{p}=0.5222)$, the medication groups were combined for subsequent analysis.

\section{Patient characteristics}

Mean age (SD) of the study population was 62.7 (10.6) years. $42.6 \%$ were female. $28.9 \%$ of patients had a history of macrovascular complications, while $16.4 \%$ had a history of microvascular complications. Further details are given in table 1 . Average score (SD) of the Worry Scale of HFS-II for the entire sample was 8.06 (10.4), with a minimum of 0 and a maximum of 51 . While 125 patients were extremely satisfied with their medication (TSQM question on treatment satisfaction), 113 patients, 100 patients, and 44 patients reported being 'very satisfied', 'satisfied', and 'somewhat satisfied' with their medication, respectively. The number of patients who were 'dissatis-

Table I: Patient characteristics

\begin{tabular}{lc}
\hline & Mean (SD) \\
\hline Age, years & $62.7(10.6)$ \\
Gender & \\
Female & $42.6 \%$ \\
Male & $57.4 \%$ \\
Married & $62.8 \%$ \\
$\mathrm{HBA}_{\text {Ic }}$ & $7.24(1.23)$ \\
$\mathrm{HBA}_{\text {Ic }}$ at goal $[6.5 \%]$ & $29.1 \%$ \\
Weight $(\mathrm{kg})^{\mathrm{B} \text { MI }\left(\mathrm{kg} / \mathrm{m}^{2}\right)}$ & $87.3(15.8)$ \\
History of complications & $29.6(4.6)$ \\
Macrovascular & \\
Microvascular & $28.9 \%$ \\
Duration of diabetes, years & $16.4 \%$ \\
$<4$ & \\
$4-6$ & $15.1 \%$ \\
$7-9$ & $24.6 \%$ \\
$>9$ & $31.7 \%$ \\
& $28.6 \%$ \\
\hline
\end{tabular}


fied', 'very dissatisfied', and 'extremely dissatisfied' were only 4,5 , and 1 , respectively.

\section{Construction of MID}

MIDs determined according to distribution-based methods varied widely (see table 2 ). While the MID for the Worry Scale of HFS-II based on the standard error of measurement was 2.0, MID based on $8 \%$ of the theoretical score range was 5.8. MIDs based on 0.2, 0.30, 0.33 and 0.5 multiplied by standard deviation, respectively, varied between 2.1 and 5.2 for the Worry Scale of HFS-II.

Mean score (+/- SD, $\mathrm{n}=$ number of patients in category) of the Worry Scale of HFS-II by satisfaction with treatment was $5.3(+/-7.0, \mathrm{n}=125)$ for patients who were extremely satisfied, $7.6(+/-9.4, \mathrm{n}=113)$ for patients who were very satisfied, $9.4(+/-11.7, \mathrm{n}=100)$ for patients who were satisfied, $13.5(+/-14.4, \mathrm{n}=44)$ for patients who were somewhat satisfied, $16.5(+/-13.0, \mathrm{n}=4)$ for patients who were dissatisfied, $8.4(+/-12.8, \mathrm{n}=5)$ for patients who were very dissatisfied, and 2.0 for a single patient who was extremely dissatisfied. Comparing mean scores of the Worry Scale of HFS-II for patients who were less than satisfied with patients who were satisfied resulted in an MID of 3.6 (see figure 1 ).

Using the TSQM questions on side effects (questions 6 and 8) resulted in a MID for the HFS-II Worry Scale of 3.6 and 3.9, respectively. The MIDs are based on the answers of 29 patients who were 'somewhat' affected vs. 12 patients who were 'a great deal' or 'quite a bit' affected for TSQM question 6 and on 15 vs. 31 patients for TSQM question 8.

\section{Severity and fear of hypoglycaemia}

112 patients (28.6\% of total sample) reported episodes of hypoglycaemia during the previous 6 months. While 51 and 46 patients reported mild and moderate episodes of hypoglycaemia, 9 and 6 patients reported severe and very severe episodes of hypoglycaemia, respectively. Thus among those reporting hypoglycaemia, $86.6 \%$ reported hypoglycaemia without the need for treatment assistance, while $13.4 \%$ reported hypoglycaemia with need for assistance. The mean Worry Scale of HFS-II was 6.0 (SD 8.2, $95 \%$ CI $[5.0 ; 6.9])$ for patients who did not report hypoglycaemic episodes during the previous 6 months, and 13.3 (SD 13.4, 95\% CI [10.8; 15.8]) for patients who reported hypoglycaemia during the previous 6 months. The crude difference in the Worry Scale of HFS-II between patients who reported no hypoglycaemia and hypoglycaemia without need for treatment assistance was $6.1(\mathrm{p}=$ 0.0001 , Cohen's $d=-0.65$ [SD 0.12]). HFS-II scores were also higher in patients who reported hypoglycaemia with need for assistance compared to those who reported hypoglycaemia without need for assistance ( $\mathrm{p}=0.0100$, Cohen's $d=-0.74$ [SD 0.28]). The size of these effects was generally larger than the MIDs determined by anchor- or distribution-based methods (see figure 2).

\section{Discussion}

HFS-II scores in this study and for this patient group reflected the expected patterns in fear of hypoglycaemia. Patients who reported hypoglycaemia showed more fear than those who did not, and patients who reported more severe episodes of hypoglycaemia showed more fear that those who reported less severe episodes. Based on the MID results of this study, the difference in the Worry Scale of the HFS-II between not having and having reported hypoglycaemia without the need for assistance appears to be clinically meaningful to patients. This is particularly relevant because the vast majority of episodes of hypoglycaemia reported in this study were those which patients managed themselves without the need for assistance from others.

To determine MID, two classes of methods have been discussed in the literature, distribution-based methods and anchor-based methods [34]. Distribution-based methods are based on mathematical calculations that involve standard deviation, score range or Cronbach's alpha.

Table 2: Distribution- and anchor-based MIDs for the Worry Scale of HFS-II.

\begin{tabular}{lc}
\hline Method & MID for HFS-II \\
\hline Distribution-based MIDs & 2.1 \\
$\quad$ Standard deviation multiplied by 0.2 & 3.1 \\
Standard deviation multiplied by 0.3 & 3.4 \\
Standard deviation multiplied by 0.33 & 5.2 \\
Standard deviation multiplied by 0.5 & 5.8 \\
8\% of theoretical score range & 2.0 \\
Standard error of measurement & 3.6 \\
Anchor-based MIDs & 3.6 \\
Satisfaction with medication (TSQM question 14) & 3.9 \\
Impact of side effects on physical health (TSQM question 6) & \\
Impact of side effects on satisfaction with medication (TSQM question 8) & \\
\hline
\end{tabular}




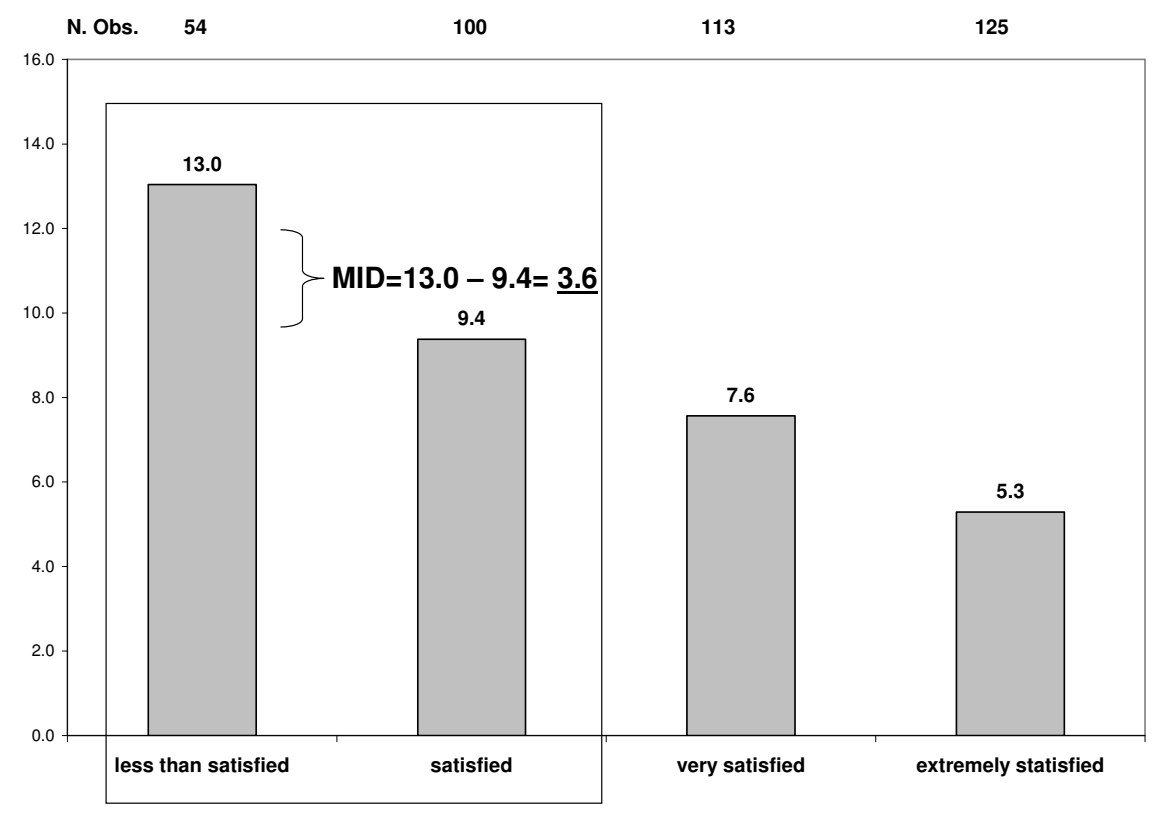

Figure I

Mean score of the Worry Scale of HFS-II, by TSQM question I4 on treatment satisfaction, and definition of an anchor-based MID.

Anchor-based methods are based on judgment of treatment success [35]. Among the group of anchor-based methods, either physician- or patient-based anchors can be applied. While distribution-based methods that involve calculations with the standard deviation, also depend on the heterogeneity of the study population [23], anchor-based methods critically depend on the validity of patients' rating [23] and on choosing response categories that reflect the importance of a change. No gold standard for determining MID currently exists. Therefore we followed the suggestion of Guyatt et al. 2002 that is more accurately to report a range of MID retrieved by different approaches than to recommend a single MID.

Wells et al., had patients compare their own health status with that of their peers. MID was calculated from the difference in score between patients who felt 'somewhat better than other patients' and patients who felt 'about the same' [32]. Another approach is to have patients rate their own improvement due to treatment. Depending on the disease, MID is then calculated as the difference in mean scores between patients who report a 'small improvement' and those who felt 'a little worse', or between patients who report a 'small improvement' and those who reported 'no change' $[22,23,29,36]$. Walters and Brazier surveyed change in condition over time to establish a MID for quality of life measures, calculated as the changes in score means between patients reporting 'an improvement' and those reporting 'no change' in condition [31]. In our study, one of the anchors chosen to determine MID was based on treatment satisfaction ('less than satisfied' vs. 'satisfied') which we considered closely related to the construct of self-reported treatment success ('no change' vs. 'small improvement') frequently recommended for studies that examine treatment effects $[27,30]$.

The results for the MID using TSQM question 14 on treatment satisfaction (3.6) and TSQM questions 6 and 8 on side effects (3.6 and 3.9) were relatively consistent. Anchor-based MID estimates of the HFS-II were well within the range obtained from distribution-based methods. However, compared to the MID based on treatment satisfaction $(\mathrm{n}=154)$, the MID's based on side effects were derived from the smaller number of patients $(n=29)$ who reported side effects. Also, hypoglycaemia does not appear to have been the only source for variation in treatment satisfaction. Hypoglycaemia may not have been the only side effect experienced by patients. For these reasons, these results should be considered exploratory. The unexpected low HFS scores for patients who were 'very dissatisfied' (5 patients, HFS-II 12.8) and 'extremely dissatisfied' (1 patient, HFS-II 2.0) compared to patients who were 'dissatisfied' (4 patients, HFS-II 16.5) or 'somewhat satisfied' (44 patients, HFS-II 13.5) may be due in part to the low number of patients in the categories. Another explanation is that perhaps these patients did not adhere to 


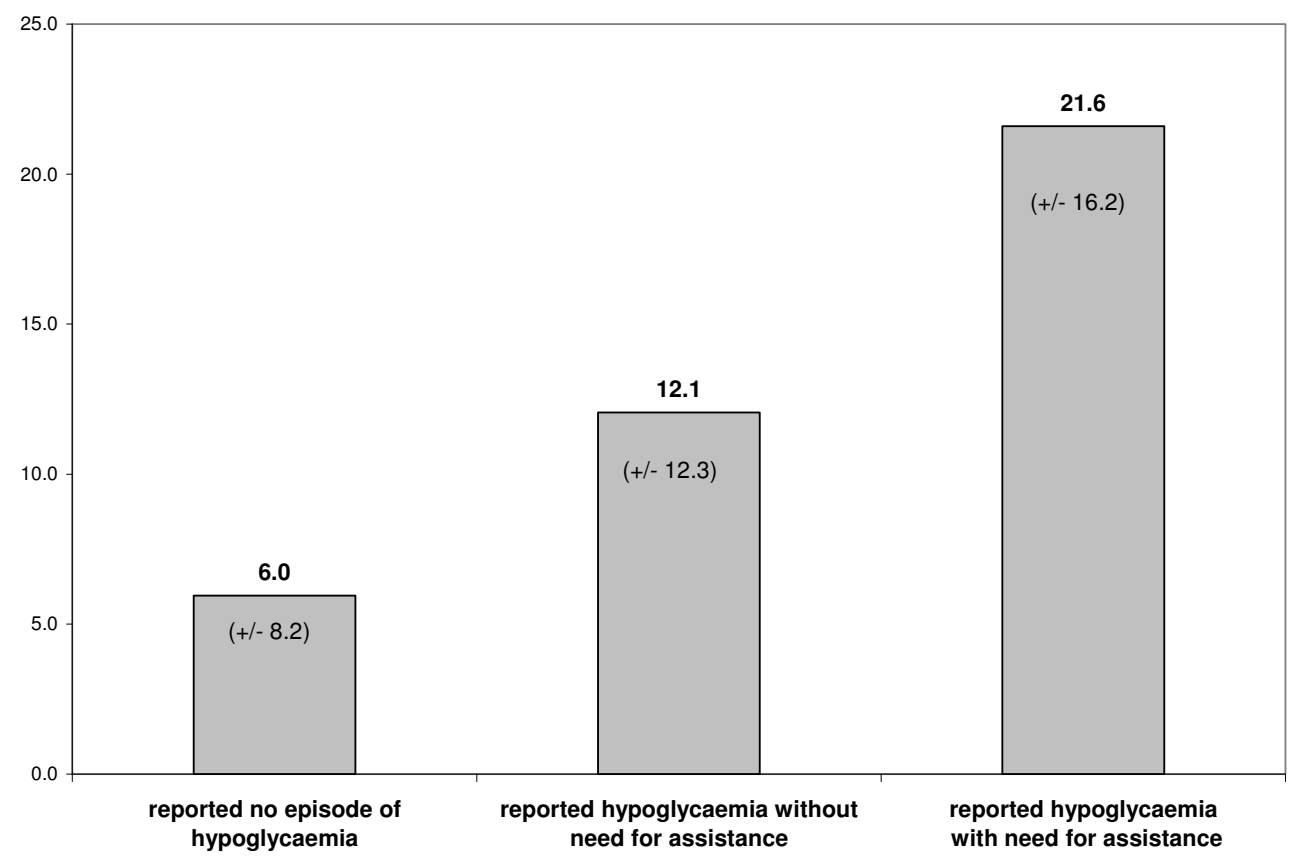

\section{Figure 2}

Mean score (+/- SD) of the Worry Scale of HFS-II, by severity of hypoglycaemia.

their prescribed medication regimen due to their dissatisfaction and, therefore, had less risk of hypoglycaemia.

While the response categories 'extremely dissatisfied', 'very dissatisfied', 'dissatisfied', and 'somewhat satisfied' were aggregated to 'less than satisfied', we did not aggregate the other response categories of the TSQM question on treatment satisfaction. In our opinion, aggregating 'satisfied', 'very satisfied', and 'extremely satisfied' and comparing these patients to patients 'less than satisfied' would have no longer constituted a minimum clinically important difference [27].

It is important to note, however, that our MID estimates may not apply to other types of patients with diabetes [37]. Patients in this study had type 2 diabetes managed by combined oral anti-hyperglycemic medications, and the majority of them $(71 \%)$ reported no hypoglycaemic episodes in the previous 6 months. Less than $4 \%$ reported severe or very severe episodes. Different MID estimates would likely be generated in the subgroup of patients with type 1 diabetes who experience frequent, recurrent episodes of severe hypoglycaemia. Nonetheless, the results of this study suggest that fear of hypoglycaemia, as measured by the HFS-II, can be a useful outcome variable in diabetes health services research, and that even relatively small differences in scores can be clinically meaningful to patients with type 2 diabetes mellitus using oral anti-hyperglycemic medications.

Observational studies can provide valuable information on effectiveness due to real-world settings and larger study populations $[38,39]$. However, self-reported outcomes from a large number of sites also introduce bias and limitations. The participating physicians may not have always had complete knowledge about parallel prescriptions to their patients and patient's visits to other physicians or hospitals. Eventually, this might have led to incomplete data on patient's medical history, or inclusion of patients who would have otherwise been excluded. Episodes of hypoglycaemia were most likely underreported in our study population, since many patients with diabetes may not always recall or recognize symptoms of hypoglycaemia $[6,10,17]$, or may have limited knowledge about hypoglycaemia itself [40].

\section{Conclusion}

The methodological approach suggested in this study might also be applicable to other patient reported outcomes, in particular, when the MID cannot be based on treatment success. By using the concept of MID, it could be shown that the difference between having reported no hypoglycaemia and having reported hypoglycaemia without need for assistance is clinically meaningful to patients with type 2 diabetes mellitus on oral anti-hyperglycaemic 
agents. Whether the MID estimates for HFS scores found in this study are applicable to different types of diabetes patients, countries, and cultures should be subject of future research.

\section{Conflict of interests statement}

TS was on a research fellowship sponsored by Merck \& Co., Inc, by the time the article was written. LGF has been working under a consultancy agreement for Merck \& Co., Inc. LGF has also worked under consultancy agreements or received research grants from Abbott Diabetes Care, Abbott Labs. KJK and CA are employees of Merck \& Co., Inc.

\section{Authors' contributions}

KJK conceived the idea to write this paper. TS analyzed the data and drafted the first version of the manuscript. All authors contributed to the conception and design of the study, to interpreting the data, and to writing the manuscript. All authors read and approved the final manuscript.

\section{References}

I. Cook MN, Girman CJ, Stein PP, Alexander CM, Holman RR: Glycemic control continues to deteriorate after sulfonylureas are added to metformin among patients with type 2 diabetes. Diabetes Care 2005, 28:995-1000.

2. Nathan DM, Buse JB, Mayer BD, Heine RJ, Holman RR, Sherwin R, et al.: Management of hyperglycemia in type 2 diabetes: a consensus algorithm for the initiation and adjustment of therapy. Diabetes Care 2006, 29:1963-1972.

3. Cook MN, Girman CJ, Stein PP, Alexander CM: Initial monotherapy with either metformin or sulphonylureas often fails to achieve or maintain current glycaemic goals in patients with type 2 diabetes in UK primary care. Diabet Med 2007, 24:350-358.

4. Home PD, Jones NP, Pocock SJ, Beck-Nielsen H, Gomis R, Hanefeld $M$, et al.: Rosiglitazone RECORD study: glucose control outcomes at 18 months. Diabet Med 2007, 24:626-634.

5. Charbonnel B, Schernthaner G, Brunetti P, Matthews DR, Tan MH, Hanefeld M: Long-term efficacy and tolerability of add-on pioglitazone therapy to failing monotherapy compared with addition of gliclazide or metformin in patients with type 2 diabetes. Diabetologia 2005, 48: 1093-I I04.

6. Chelliah A, Burke MR: Hypoglycemia in elderly patients with diabetes mellitus. Drugs \& Aging 2004, 2 I:5 I I-530.

7. Davis S, Alonso MD: Hypoglycemia as a barrier to glycemic control. J Diabetes Complications 2004, 18:60-68.

8. Amiel SA, Dixon T, Mann R, Jameson K: Hypoglycaemia in type 2 diabetes. Diabet Med 2008, 25:245-254.

9. Banarer S, Cryer PE: Gypoglycemia in type 2 diabetes. The Medical Clinics of North America 2004, 88: I I07-III 6.

10. Boyle PJ, Zrebiec J: Physiological and behavioral aspects of glycemic control and hypoglycemia in diabetes. South Med J 2007, 100:175-182.

II. Murata GH, Duckworth WC, Shah JH, Wendel CS, Mohler MJ, Hoffmann RM: Hypoglycemia in stable, insulin-treated verterans with type 2 diabetes, a retrospective study of 1662 episodes. J Diabetes Complications 2005, 19:10-I7.

12. Zammitt NN, Frier BM: Hypoglycemia in type 2 diabetes. Diabetes Care 2005, 28:2948-296I.

13. Frier BM: Hypoglycemia and cognitive function in diabetes. Int J Clin Pract 200I, SI 23:30-37.

14. Rosenthal JM, Amiel SA, Yágüez L, Bullmore E, Hopkins D, Evans M, et al:: The effect of acute hypoglycemia on brain function and activation. Diabetes 200I, 50:1618-1626.

15. Whitmer RA, Karter AJ, Yaffe K, Quesenberry CP Jr, Selby JV: Hypoglycemic episodes and risk of dementia in older patients with type 2 diabetes mellitus. JAMA 2009, 30I:1565-1572

16. American Diabetes Association Workgroup on Hypoglycemia: Defining and reporting hypoglycemia in diabetes. Diabetes Care 2005, 28: I245-1249.

17. Boyle PJ, Zrebiec J: Management of diabetes-related hypoglycemia. South Med J 2007, 100:183-194.

18. Nattrass M, Lauritzen T: Review of prandial glucose regulation with reaglinide: a solution to the problem of hyoglycemia in the treatment of type 2 diabetes. Int J Obes 2000, 24:2I-3I.

19. Gonder-Frederick L, Cox DJ, Clarke W, Julian D: Blood glucose awareness training. In Psychology in diabetes Care Edited by: Snoek FJ, Skinner TC. New York: Wiley; 2000:169-206.

20. Cox DJ, Gonder-Frederick L, Polonsky W, Schlundt D, Kovatchev B, Clarke W: Blood glucose awareness training (BGAT-2): Longterm benefits. Diabetes Care 200I, 24:638-42.

21. Johnson JA, Kotovych M, Ryan EA, Shapiro AM: Reduced fear of hypoglycemia in successful islet transplantation. Diabetes Care 2004, 27:624-25.

22. Jaeschke R, Singer J, Guyatt DH: Measurement of health status: ascertaining the minimal clinically important difference. Control Clin Trials 1989, 10:407-4I5.

23. Guyatt GH, Osoba D, Wu AW, Wyrwich KW, Norman GR, the clinical significance consensus meeting group: Methods to explain the clinical significance of health status measures. Mayo Clin Proc 2002, 77:37I-383.

24. Atkinson MJ, Sinha A, Hass SL, Colman SS, Kumar RN, Brod M, et al.: Validation of a general measure of treatment satisfaction, the Treatment Satisfaction Questionaire for Medication (TSQM), using a national panel study of chronic disease. Health and Quality of Life Outcomes 2004, 2: I-13.

25. Cox DJ, Irvine A, Gonder-Frederick L, Nowacek G, Butterfield J: Fear of hypoglycemia: quantification, validation and utilization. Diabetes Care 1987, 10:617-621.

26. Irvine $A$, Cox DJ, Gonder-Frederick L: The Fear of hypoglycemia scale. In Handbook of psychology and diabetes: a guide to psychological measurement and diabetes research and practice Edited by: Bradley $\mathrm{C}$. Langhorne: Hardwood Academia Publishing; 1994:133-158.

27. Revicki DA, Hays RD, Cella D, Sloan JA: Recommended methods for determining responsiveness and minimally important differences for patient-reported outcomes. J Clin Epidemiol 2008, 61:102-9.

28. U.S. Department of Health and Human Services FDA Center for Drug Evaluation and Research, U.S. Department of Health and Human Services FDA Center for Biologics Evaluation and Research, U.S. Department of Health and Human Services FDA Center for Devices and Radiological Health: Guidance for industry: patient-reported outcome measures: use in medical product development to support labelling claims. Health and Quality of Life Outcomes 2006, 4:I-20.

29. Yost KJ, Cella D, Chawla A, Holmgren E, Eton DT, Ayanian JZ, et al:: Minimally important differences were estimated for the Functional Assessment of Cancer Therapy-Colorectal (FACT-C) instrument using a combination of distributionand anchor-based approaches. J Clin Epidemiol 2005, 58: $124|-| 25 \mid$.

30. Beaton D, Bombardier C, Katz JN, Wright JG, Wells GA, Boers M, et al.: Looking for important change/differences in studies of responsiveness. The Journal of Rheumatology 200I, 28:400-405.

31. Walters SJ, Brazier JE: Comparison of the minimally important difference for two health state utility measures: EQ-5D and SF-6d. Qual Life Res 2005, I 4:1523-I532.

32. Wells GA, Tugwell P, Kraag GR, Baker PRA, Groh J, Redelmeier DA: Minimum important difference between patients with rheumatoid arthitis: the patient's perspective. The Journal of Rheumatology 1993, 20:557-560.

33. Leese GP, Wang J, Broomhall J, Kelly P, Marsden A, Morrison W, et al.: Frequency of severe hypoglycemia requiring emergency treatment in type 1 and type 2 diabetes. Diabetes Care 2003, 26: II76-II80.

34. Lydick E, Epstein R: Interpretation of quality of life changes. Qual Life Res 1993, 2:221-6.

35. Marquis $\mathrm{P}$, Chassany $\mathrm{O}$, Abetz L: A comprehensive strategy for the interpretation of quality-of-life data based existing methods. Value in Health 2004, 7:93-104. 
36. Wells GA, Beaton D, Shea B, Boers M, Simon L, Strand V, et al:: Minimal clinically important differences: review of methods. The Journal of Rheumatology 200I, 28:406-4I2.

37. Norquist J, Girman C, Santanello N: Some considerations for the interpretation of health-related quality of life data. Value in Health 2005, 8:80-81.

38. Riedel A, Heien H, Wogen J, Plauschinat C: Loss of glycemic control in patients with type 2 diabetes mellitus who were receiving initial metformin, sulfonylurea, or thiazolidinedione monotherapy. Pharmacotherapy 2007, 27: I 102-1 II0.

39. McKee M, Brotton A, Black N, McPherson K, Sanderson C, Bain C: Methods in health services research: Interpreting the evidence: choosing between randomised and non-randomised studies. BMJ 1999, 319:312-315.

40. Murata GH, Hoffmann RM, Shah JH, Wendel CS, Duckworth WC: A probabilistic model for predicting hypoglycemia in type 2 diabetes mellitus. Arch Intern Med 2004, 164: 1445-I450.

Publish with Bio Med Central and every scientist can read your work free of charge

"BioMed Central will be the most significant development for disseminating the results of biomedical research in our lifetime. "

Sir Paul Nurse, Cancer Research UK

Your research papers will be:

- available free of charge to the entire biomedical community

- peer reviewed and published immediately upon acceptance

- cited in PubMed and archived on PubMed Central

- yours - you keep the copyright

Submit your manuscript here:

http://www.biomedcentral.com/info/publishing_adv.asp 